\title{
Community Pediatric Hospitalist Workload: Results from a National Survey
}

\author{
Francisco Alvarez, MD* , Corrie E McDaniel, DO², Krista Birnie, MD³, Craig Gosdin, MD, MSHA4, Allison Mariani, MD,6,

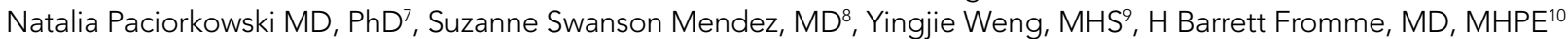

\begin{abstract}
${ }^{1}$ Division of Pediatric Hospital Medicine, Stanford School of Medicine, Stanford, California; ${ }^{2}$ University of Washington School of Medicine, Seattle Children's Hospital, Seattle, Washington; ${ }^{3}$ Pediatric Hospital Medicine Department, Tufts University School of Medicine, Boston, Massachusetts; ${ }^{4}$ Department of Hospital Medicine, University of Cincinnati College of Medicine, Cincinnati Children's Hospital Medical Center, Cincinnati, Ohio; ${ }^{5}$ Pediatric Hospital Medicine Department, Tufts University School of Medicine, Boston, Massachusetts; ${ }^{6}$ Pediatrics Department, Newton-Wellesley Hospital, Newton, Massachusetts; ${ }^{7}$ Pediatric Hospitalist Service, Rochester General Hospital, Rochester, New York; ${ }^{8}$ Division of Pediatrics and Neonatology, St. Charles Medical Center, Bend, Oregon; ${ }^{9}$ Quantitative Sciences Unit, Stanford School of Medicine, Stanford, California; ${ }^{10}$ University of Chicago, Pritzker School of Medicine, Chicago, Illinois.
\end{abstract}

As a newly recognized subspecialty, understanding programmatic models for pediatric hospital medicine (PHM) programs is vital to lay the groundwork for a sustainable field. Although variability has been described within university-based PHM programs, there remains no national benchmark for community-based PHM programs. In this report, we describe the workload, clinical services, employment, and perception of sustainability of 70 community-based PHM programs in 29 states through a survey of community site leaders. The median hours for a full-time hospitalist was 1,882 hours/year with those employed by community hospitals working $8 \%$ more hours/ year and viewing appropriate morning pediatric census as $20 \%$ higher than those employed by university institutions. Forty-three out of $70(63 \%)$ site leaders perceived their programs as sustainable, with no significant difference by employer structure. Future studies should further explore root causes for workload discrepancies between community and academic employed programs along with establishing potential standards for PHM program development. Journal of Hospital Medicine 2019;14:682-685. () 2019 Society of Hospital Medicine s a newly recognized specialty, pediatric hospital medicine (PHM) continues to expand and diversify. ${ }^{1}$ Pediatric hospitalists care for children in hospitals ranging from small, rural community hospitals to large, free-standing quaternary children's hospitals. ${ }^{2-4}$ In addition, more than $10 \%$ of graduating pediatric residents are seeking future careers within PHM. ${ }^{5}$

In 2018, Fromme et al. published a study describing clinical workload for pediatric hospitalists within university-based settings. ${ }^{6}$ They characterized the diversity of work models and programmatic sustainability but limited the study to university-based programs. With over half of children receiving care within community hospitals, ${ }^{7}$ workforce patterns for community-based pediatric hospitalists should be characterized to maximize sustainability and minimize attrition across the field.

In this study, we describe programmatic variability in clinical work expectations of 70 community-based PHM programs. We aimed to describe existing work models and expectations of community-based program leaders as they relate to their unique clinical setting.

*Corresponding Author: Francisco Alvarez, MD; E-mail: falvare1@stanford. edu; Telephone: 650-736-4421

Published online first August 21, 2019.

Find Additional Supporting Information in the online version of this article.

Received: April 19, 2019; Revised: June 5, 2019; Accepted: June 5, 2019

๑ 2019 Society of Hospital Medicine DOI 10.12788/jhm.3263

\section{METHODS}

We conducted a cross-sectional survey of community-based PHM site directors through structured interviews. Community hospital programs were self-defined by the study participants, although typically defined as general hospitals that admit pediatric patients and are not free-standing or children's hospitals within a general hospital. Survey respondents were asked to answer questions only reflecting expectations at their community hospital.

\section{Survey Design and Content}

Building from a tool used by Fromme et al. ${ }^{6}$ we created a 12-question structured interview questionnaire focused on three areas: (1) full-time employment (FTE) metrics including definitions of a 1.0 FTE, "typical" shifts, and weekend responsibilities; (2) work volume including census parameters, service-line coverage expectations, back-up systems, and overnight call responsibilities; and (3) programmatic model including sense of sustainability (eg, minimizing burnout and attrition), support for activities such as administrative or research time, and employer model (Appendix).

We modified the survey through research team consensus. After pilot-testing by research team members at their own sites, the survey was refined for item clarity, structural design, and length. We chose to administer surveys through phone interviews over a traditional distribution due to anticipated variability in work models. The research team discussed how each question should be asked, and responses were clarified to maintain consistency. 


\section{Survey Administration}

Given the absence of a national registry or database for community-based PHM programs, study participation was solicited through an invitation posted on the American Academy of Pediatrics Section on Hospital Medicine (AAP SOHM) Listserv and the AAP SOHM Community Hospitalist Listserv in May 2018. Invitations were posted twice at two weeks apart. Each research team member completed 6-19 interviews. Responses to survey questions were recorded in REDCap, a secure, webbased data capture instrument. ${ }^{8}$

Participating in the study was considered implied consent, and participants did not receive a monetary incentive, although respondents were offered deidentified survey data for participation. The study was exempted through the University of Chicago Institutional Review Board.

\section{Data Analysis}

Employers were dichotomized as community hospital employer (including primary community hospital employment/private organization) or noncommunity hospital employer (including children's/university hospital employment or school of medicine). Descriptive statistics were reported to compare the demographics of two employer groups. $P$ values were calculated using two-sample t-tests for the continuous variables and chi-square or Fisher-exact tests for the categorical variables. Mann-Whitney U-test was performed for continuous variables without normality. Analyses were performed using the R Statistical Programming Language (R Foundation for Statistical Computing, Vienna, Austria), version 3.4.3.

\section{RESULTS}

\section{Participation and Program Characteristics}

We interviewed 70 community-based PHM site directors representing programs across 29 states (Table 1) and five geographic regions: Midwest (34.3\%), Northeast (11.4\%), Southeast (15.7\%), Southwest (4.3\%), and West (34.3\%). Employer models varied across groups, with more noncommunity hospital employers (57\%) than community hospital employers (43\%). The top three services covered by pediatric hospitalists were pediatric inpatient or observation bed admissions (97\%), emergency department consults (89\%), and general newborns (67\%). PHM programs also provided coverage for other services, including newborn deliveries (43\%), Special Care Nursery/Level II Neonatal Intensive Care Unit (41\%), step-down unit (20\%), and mental health units (13\%). About $59 \%$ of programs provided education for family medicine residents, $36 \%$ were for pediatric residents, and $70 \%$ worked with advanced practice providers. The majority of programs (70\%) provided in-house coverage overnight.

\section{Clinical Work Expectations and Employer Model}

Clinical work expectations varied broadly across programs (Table 2). The median expected hours for a 1.0 FTE was 1,882 hours per year (interquartile range $[I Q R] 1,805,2,016$ ), and the median expected weekend coverage/year (defined as covering two days or two nights of the weekend) was 21 (IQR 14,
TABLE 1. Description of Community-based Pediatric Hospital Medicine Programs

\begin{tabular}{|c|c|}
\hline Total Number of Hospitals & $\begin{array}{c}70 \\
n(\%)\end{array}$ \\
\hline \multicolumn{2}{|l|}{ Employer of the pediatric hospitalist staff } \\
\hline Children's/university hospital & $31(44)$ \\
\hline Regional/ community hospital & $17(24)$ \\
\hline Private organization & $13(19)$ \\
\hline School of medicine & $5(7)$ \\
\hline Independent company contracted by children's/university hospital & $4(6)$ \\
\hline \multicolumn{2}{|l|}{ Services Covered } \\
\hline Pediatric floor patients (inpatient and observation) & $68(97)$ \\
\hline ED consults & $62(89)$ \\
\hline General newborn nursery/Level I NICU & $47(67)$ \\
\hline Newborn deliveries & $30(43)$ \\
\hline Special care nursery/Level II NICU & $29(41)$ \\
\hline Circumcision & $17(24)$ \\
\hline Step-down unit & $14(20)$ \\
\hline PICU care & $11(16)$ \\
\hline Sedation & $10(14)$ \\
\hline Mental health units & $9(13)$ \\
\hline ED/urgent care primary coverage & $4(6)$ \\
\hline Level III NICU & $1(1)$ \\
\hline Other & $16(23)$ \\
\hline \multicolumn{2}{|l|}{ Presence of residents and advanced practice providers } \\
\hline Advanced practice providers (PA, NP) & $49(70)$ \\
\hline Family medicine residents & $23(59)$ \\
\hline Pediatric residents & $14(36)$ \\
\hline Attending 24-hour in-house coverage & $49(70)$ \\
\hline $\begin{array}{l}\text { TOTAL program \% support for nondirect patient care activi } \\
\text { [IQR] }\end{array}$ & $4[0,10]$ \\
\hline
\end{tabular}

Program perceived as sustainable

$44(63)$

Abbreviatons: $E D$, emergency department; $I Q R$, interquartile range; $N P$, nurse practioner; NICU, neonatal intensive care unit; PA, physician assistant; PHM, pediatric hospital medicine; $\mathrm{PICU}$, pediatric intensive care unit.

24). Most programs did not expand staff coverage based on seasonality $(73 \%)$, and less than $20 \%$ of programs operated with a census cap. Median support for nondirect patient care activities was $4 \%(I Q R), 10)$ of a program's total FTE (ie, a 5.0 FTE program would have 0.20 FTE support). Programs with community hospital employers had an $8 \%$ higher expectation of 1.0 FTE hours/year $(P=.01)$ and viewed an appropriate pediatric morning census as $20 \%$ higher $(P=.01$; Table 2$)$.

\section{Program Sustainability}

Twenty-six (37\%) site directors described their program as unsustainable. When programmatic characteristics and clinical work expectations were analyzed by perception of sustainability, we observed no difference between programs that were perceived as unsustainable in the number of 1.0 FTE hours/ year $(P=.16)$, weekends/year $(P=.65)$, in-house call $(P=.36)$, or the presence of a back-up system $(P=.61)$. 
TABLE 2. Comparison of Programmatic Expectations

\begin{tabular}{|c|c|c|c|c|}
\hline & All & $\begin{array}{c}\text { Community } \\
\text { Hospital Employer }\end{array}$ & $\begin{array}{l}\text { Noncommunity Hospital } \\
\text { Employer }\end{array}$ & $P$ Value \\
\hline Number of Hospitals & 70 & 30 & 40 & \\
\hline \multicolumn{5}{|l|}{ Metric used to describe FTE, $n$ (\%) } \\
\hline Weeks & $4(5.7)$ & $2(6.7)$ & $2(5.0)$ & - \\
\hline Shifts & $21(30.0)$ & $12(40.0)$ & $9(22.5)$ & - \\
\hline Hours & $44(62.9)$ & $16(53.3)$ & $28(70.0)$ & - \\
\hline Others (days) & $1(1.4)$ & $(0.0)$ & $1(2.5)$ & - \\
\hline \multirow[t]{2}{*}{ 1.0 FTE in hours per year, median (IQR) } & 1,882 & 1,964 & 1,872 & .01 \\
\hline & {$[1,805,2,016]$} & {$[1,825,2,122]$} & {$[1,794,1,948]$} & \\
\hline Weekends total/ year in $1.0 \mathrm{FTE}$, median (IQR) & $21[14,24]$ & $22[16,24]$ & $21[14,24]$ & .58 \\
\hline Pager overnight, $\mathrm{n}(\%)$ & $21(30.0)$ & $11(36.7)$ & $10(25.0)$ & .43 \\
\hline \multicolumn{5}{|l|}{ Expansion of staff/coverage seasonally, $n(\%)$} \\
\hline No & $51(72.9)$ & $21(70.0)$ & $30(75.0)$ & - \\
\hline Yes & $10(14.3)$ & $5(16.7)$ & $5(12.5)$ & - \\
\hline Missing & $9(12.9)$ & $4(13.3)$ & $5(12.5)$ & - \\
\hline Back-up system formally in place, $n(\%)$ & $23(32.9)$ & $11(36.7)$ & $12(30.0)$ & .74 \\
\hline Census cap in place, $n(\%)$ & $11(15.7)$ & $3(10.0)$ & $8(20.0)$ & $.42^{\mathrm{a}}$ \\
\hline Pediatrics & $3(4.3)$ & $0(0.0)$ & $3(7.5)$ & $.35^{\mathrm{a}}$ \\
\hline Newborn & $0(0.0)$ & $0(0.0)$ & $0(0.0)$ & - \\
\hline Combined & $6(8.6)$ & $3(10.0)$ & $3(7.5)$ & $1.00^{\mathrm{a}}$ \\
\hline \multicolumn{5}{|l|}{ Morning census, median [IQR] } \\
\hline Combined & $15[12,15]$ & $15[13,15]$ & $15[12,15]$ & .85 \\
\hline Pediatric only & $12[10,14]$ & $12[12,15]$ & $10[10,12]$ & .01 \\
\hline Newborn only & $15[0,20]$ & $15[0,20]$ & $15[5,20]$ & .82 \\
\hline Sustainable, n (\%) & $44(62.9)$ & $17(56.7)$ & $27(67.5)$ & .50 \\
\hline
\end{tabular}

${ }^{\mathrm{a}}$ Fisher's exact tests were performed

Abbreviations: FTE, full-time employment; IQR, interquartile range

\section{DISCUSSION}

To our knowledge, this study is the first to describe clinical work models exclusively for pediatric community hospitalist programs. We found that expectations for clinical FTE hours, weekend coverage, appropriate morning census, support for nondirect patient care activities, and perception of sustainability varied broadly across programs. The only variable affecting some of these differences was employer model, with those employed by a community hospital employer having a higher expectation for hours/year and appropriate morning pediatric census than those employed by noncommunity hospital employers.

With a growing emphasis on physician burnout and career satisfaction, ${ }^{9-11}$ understanding the characteristics of community hospital work settings is critical for identifying and building sustainable employment models. Previous studies have identified that the balance of clinical and nonclinical responsibilities and the setting of community versus university-based programs are major contributors to burnout and career satisfaction. .,11 $^{-11}$ Interestingly, although community hospital-based programs have limited FTE for nondirect patient care activities, we found that a higher percentage of program site directors perceived their program models as sustainable when compared with university-based programs in prior research (63\% versus 50\%). ${ }^{6}$ Elucidating why community hospital PHM programs are perceived as more sustainable provides an opportunity for fu- 
ture research. Potential reasons may include fewer academic requirements for promotion or an increased connection to a local community.

We also found that the employer model had a statistically significant impact on expected FTE hours per year but not on perception of sustainability. Programs employed by community hospitals worked $8 \%$ more hours per year than those employed by noncommunity hospital employers and accepted a higher morning pediatric census. This variation in hours and census level appropriateness is likely multifactorial, potentially from higher nonclinical expectations for promotion (eg, academic or scholarly production) at school of medicine or children's hospital employed programs versus limited reimbursement for administrative responsibilities within community hospital employment models.

There are several potential next steps for our findings. As our data are the first attempt (to our knowledge) at describing the current practice and expectations exclusively within community hospital programs, this study can be used as a starting point for the development of workload expectation standards. Increasing transparency nationally for individual community programs potentially promotes discussions around burnout and attrition. Having objective data to compare program models may assist in advocating with local hospital leadership for restructuring that better aligns with national norms.

Our study has several limitations. First, our sampling frame was based upon a self-selection of program directors. This may have led to a biased representation of programs with higher workloads motivated to develop a standard to compare with other programs, which may have potentially led to an overestimation of hours. Second, without a registry or database for community-based pediatric hospitalist programs, we do not know the percentage of community-based programs that our sample represents. Although our results cannot speak for all community PHM programs, we attempted to mitigate nonresponse bias through the breadth of programs represented, which spanned 29 states, five geographic regions, and teaching and nonteaching programs. The interview-based method for data collection allowed the research team to clarify questions and responses across sites, thereby improving the quality and consistency of the data for the represented study sample. Finally, other factors possibly contributed to sustainability that we did not address in this study, such as programs that are dependent on billable encounters as part of their salary support.

\section{CONCLUSION}

As a newly recognized subspecialty, creating a reference for community-based program leaders to determine and discuss individual models and expectations with hospital administrators may help address programmatic sustainability. It may also allow for the analysis of long-term career satisfaction and longevity within community PHM programs based on workload. Future studies should further explore root causes for workload discrepancies between community and university employed programs along with establishing potential standards for PHM program development.

\section{Acknowledgments}

We would like to thank the Stanford School of Medicine Quantitative Sciences Unit staff for their assistance in statistical analysis.

Disclosure: The authors have nothing to disclose.

\section{References}

1. Robert MW, Lee G. Zero to 50,000 -The 20th anniversary of the hospitalist. $N$ Engl J Med. 2016;375(11):1009-1011. https://doi.org/10.1056/NEJMp1607958.

2. Gosdin C, Simmons J, Yau C, Sucharew H, Carlson D, Paciorkowski N. Survey of academic pediatric hospitalist programs in the US: organizational, administrative, and financial factors. J Hosp Med. 2013;8(6):285-291. https://doi. org/10.1002/jhm.2020

3. Paul DH, Jennifer D, Elizabeth $R$, et al. Proposed dashboard for pediatric hospital medicine groups. Hosp Pediatr. 2012;2(2):59-68. https://doi. org/10.1542/hpeds.2012-0004

4. Gary LF, Kathryn B, Kamilah N, Indu L. Characteristics of the pediatric hospitalist workforce: its roles and work environment. Pediatrics 2007;120(1):33-39. https://doi.org/10.1542/peds.2007-0304

5. Leyenaar JK, Frintner MP. Graduating pediatric residents entering the hospital medicine workforce. 2006-2015. Acad Pediatr. 2018;18(2):200-207. https:// doi.org/10.1016/j.acap.2017.05.001.

6. Fromme HB, Chen CO, Fine BR, Gosdin C, Shaughnessy EE. Pediatric hospitalist workload and sustainability in university-based programs: results from a national interview-based survey. J Hosp Med. 2018;13(10):702-705. https:// doi.org/10.12788/jhm.2977.

7. Leyenaar JK, Ralston SL, Shieh MS, Pekow PS, Mangione-Smith R, Lindenauer PK. Epidemiology of pediatric hospitalizations at general hospitals and freestanding children's hospitals in the United States. J Hosp Med. 2016;11(11):743-749. https://doi.org/10.1002/jhm.2624.

8. Harris PA, Taylor R, Thielke R, Payne J, Gonzalez N, Conde JG. Research electronic data capture (REDCap)--a metadata-driven methodology and workflow process for providing translational research informatics support. $J$ Biomed Inform. 2009;42(2):377-381. https://doi.org/10.1016/j.jbi.2008.08.010.

9. Laurie $A P$, Aisha BD, Mary CO. Association between practice setting and pediatric hospitalist career satisfaction. Hosp Pediatr. 2013;3(3):285-291. https:// doi.org/10.1542/hpeds.2012-0085

10. Hinami K, Whelan CT, Wolosin RJ, Miller JA, Wetterneck TB. Worklife and satisfaction of hospitalists: toward flourishing careers. J Gen Intern Med. 2011;27(1):28-36. https://doi.org/10.1007/s11606-011-1780-z.

11. Hinami K, Whelan CT, Miller JA, Wolosin RJ, Wetterneck TB. Job characteristics, satisfaction, and burnout across hospitalist practice models. J Hosp Med. 2012;7(5):402-410. https://doi.org/10.1002/jhm.1907 